Please follow these guidelines so that your manuscript may be handled expeditiously.

Nature is an international journal covering all the sciences. Contributors should therefore bear in mind those readers who work in other fields and those for whom English is a second language, and write clearly and simply, avoiding unnecessary technical terminology. Space in the journal is limited, making competition for publication severe. Brevity is highly valued. One printed page of Nature, without interruptions of the text, contains about 1,300 words.

Manuscripts are selected for publication according to editorial assessment of their suitability and reports from independent referees. They can be sent to London or Washington and should be addressed to the Editor. Manuscripts may be dealt with in either office, depending on the subject matter, and will where necessary be sent between offices by overnight, courier. All manuscripts are acknowledged on receipt but fewer than half are sent for review. Those that are not reviewed are returned as rapidly as possible so that they may be submitted elsewhere without delay. Contributors may suggest reviewers; limited requests for the exclusion of specific reviewers are usually heeded. Manuscripts are usually sent to two or three reviewers, who are chosen for their expertise rather than their geographical location. Manuscripts accepted for publication are typeset from the London office.

Nature requests authors to deposit sequence and crystallographic data in the databases that exist for this purpose, and to mention availability of these data.

Once a manuscript is accepted for publication, contributors will receive galley proofs in about 4 weeks. Nature's staff will edit manuscripts with a view to brevity and clarity, so contributors should check their proofs carefully. Manuscripts are generally published 2-3 weeks after receipt of corrected proofs. Nature does not exact page charges. Contributors receive a reprint order form with their proofs; reprint orders are processed after the manuscript is published and payment received.

\section{Categories of paper}

Review articles survey recent developments in a field. Most are commissioned but suggestions are welcome in the form of a one-page synopsis addressed to the Reviews Coordinator. Length is negotiable in advance.

Articles are research reports whose conclusions are of general interest and which are sufficiently rounded to be a substantial advance in understanding. They should not have more than 3,000 words of text (not including figure legends) or more than six display items (figures and tables) and should not occupy more than five pages of Nature.

Articles start with a heading of 50-80 words written to advertise their content in general terms, to which editors will pay particular attention. The heading does not usually contain numbers, abbreviations or measurements. The introduction to the study is contained in the first two or three paragraphs of the article, which also briefly summarize its results and implications. Articles have fewer than 50 references and may contain a few subheadings of two or three words.

Letters are short reports of outstanding novel findings whose implications are general and important enough to be of interest to those outside the field. Letters should have 1,000 or fewer words of text and four or fewer display items. The first paragraph describes, in not more than 150 words and without the use of abbreviations, the background, rationale and chief conclusions of the study for the particular benefit of non-specialist readers. Letters do not have subheadings and contain fewer than 30 references.

Commentary articles deal with issues in, or arising from, research that are also of interest to readers outside research. Some are commissioned but suggestions can be made to the commentary editor in the form of a one-page synopsis. Commentaries are normally between one and four pages of Nature.

News and Views articles inform non-specialist readers about new scientific advances, sometimes in the form of a conference report.
Most are commissioned but proposals can be made in advance to the News and Views editor.

Scientific Correspondence is for discussion of topical scientific matters, including those published in Nature, and for miscellaneous contributions. Priority is given to letters of fewer than 500 words and 5 references.

\section{Preparation of manuscripts}

All manuscripts should be typed, double-spaced, on one side of the paper only. An original and three copies are required, each accompanied by artwork. If photographs are included, four sets of originals are required; for line drawings, one set of originals and three good-quality photocopies are acceptable. Reference lists, figure legends and tables should all be on separate sheets, all of which should be double-spaced and numbered. Relevant manuscripts in press or submitted for publication elsewhere should be included with each copy of a submitted manuscript, and clearly marked as such. Revised and resubmitted manuscripts should also be clearly marked as such and labelled with their manuscript numbers.

Titles say what the paper is about with the minimum of technical terminology and in fewer than 80 characters in the case of Articles and Letters. Active verbs, numerical values, abbreviations and punctuation are to be avoided. Titles should contain one or two key words for indexing purposes.

Artwork should be marked individually and clearly with the author's name and, when known, the manuscript number. Ideally, no figure should be larger than 28 by $22 \mathrm{~cm}$. Figures with several parts are to be avoided and are permitted only if the parts are closely related, either experimentally or logically. Unlettered originals of photographs should be provided. Original artwork is returned when a manuscript cannot be published.

Protein/nucleotide sequences should ideally be in the three-letter and not the single-letter code for amino acids. One column width of Nature can accommodate 20 amino acids or 60 base pairs. Numbering of sequences should be in the left-hand margin only, with a single space between rows.

Suggestions for cover illustrations, with captions and labelled with the manuscript number, are welcome.

Colour artwork. A charge of $£ 500$ per page is made as a contribution towards the cost of reproducing colour figures. Inability to pay these costs will not prevent the publication of essential colour figures if the circumstances are explained. Proofs of colour artwork may be sent to contributors under separate cover from their galley proofs.

Figure legends should not exceed 300 words and ideally should be shorter. The figure is described first, then, briefly, the method. Reference to a method published elsewhere is preferable to a full description. Methods are not described in the text.

References are numbered sequentially as they appear in the text, followed by those in tables and finally by those in figure legends. Only papers published or in the press are numbered and included in the reference list. All other forms of reference, including unrefereed abstracts, should be cited in the text as a personal communication, manuscript submitted or in preparation. Text is not included in reference lists. References are abbreviated according to the World List of Scientific Periodicals (Butterworths, London, 1963-65). The first and last page numbers are included; reference to books should include publisher, place and date.

Abbreviations, symbols, units and greek letters should be identified the first time they are used. Acronyms should be avoided whenever possible and, if used, defined. Footnotes are not used except for changed addresses.

Acknowledgements are brief; grant and contribution numbers are not allowed.

Submission. Manuscripts can be sent to the Editor at 4 Little Essex Street, London WC2R 3LF, UK or at 1137 National Press Building, Washington, DC 20045, USA. Manuscripts or proofs sent by air courier to London should be declared as 'manuscripts' and 'value $\$ 5^{\prime}$ to prevent the imposition of import duty and value-added tax (VAT). 